\title{
OBSERVING STELLAR SURFACE STRUCTURE WITH THE ESO-VLT INTERFEROMETER
}

\author{
O. VON DER LÜHE \\ European Southern Observatory \\ Karl-Schwarzschild-Str. 2, D - 85748 Garching \\ S. SOLANKI \\ Institut für Astronomie \\ ETH Zürich \\ AND \\ TH. REINHEIMER \\ Max-Planck-Institut für Radioastronomie \\ Auf dem Hügel 69, Bonn
}

\begin{abstract}
The ESO Very Large Telescope (VLT) has the capability to coherently combine the light from four $8 \mathrm{~m}$ telescopes and from smaller Auxiliary telescopes with baselines $4 \mathrm{~m}$ and $200 \mathrm{~m}$ (VLT Interferometer or VLTI). The resulting resolution approaches 0.5 milli-arcsec at visible wavelengths and 1 milli-arcsec in the near infra-red. We estimate that about 2000 nearby bright stars can be resolved with these baselines. The surface of about 400 stars, mainly $\mathrm{K}$ and $\mathrm{M}$ giants, can be mapped with a resolution of a dozen pixels or better across the stellar surface. This resolution permits detailed studies of the structure of stellar atmospheres, their hydrodynamics and magneto-hydrodynamics. VLTI can easily resolve starspots and very likely also convection cells on an active K-type giant at $10 \mathrm{pc}$ distance. A marginal detection of the largest starspots on a very active solar-type star at the same distance may be possible, but it appears very unlikely that the surface structure on an inactive solar-type star can be imaged.

We present computer simulations of stellar surfaces to study in detail the response of the interferometer to an extended, complex source. First results which simulate the end-to-end process in a simplified manner indicate that useful maps can be produced provided that a sufficient number of baselines are combined in Earth-rotation synthesis mode. We also give arguments that high spectral resolution will prove essential to constrain reconstructed maps.
\end{abstract}




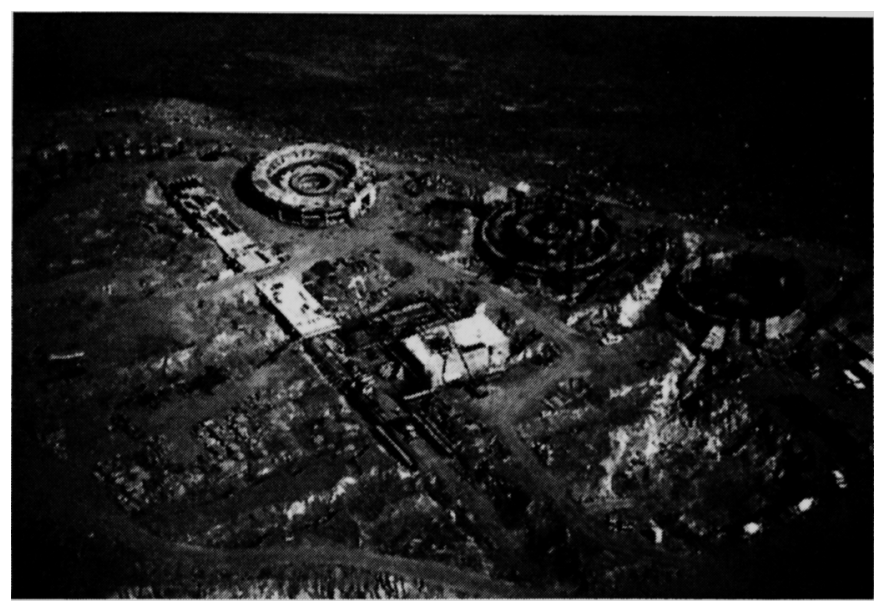

Figure 1. The VLT on Paranal April 2, 1995.

\section{Introduction}

The European Southern Observatory is currently constructing one of the largest astronomical observatories in the world; the Very Large Telescope (VLT) on Cerro Paranal in the Atacama desert in northern Chile. The VLT will ultimately include four $8 \mathrm{~m}$ Unit telescopes (UTs), three $1.8 \mathrm{~m}$ diameter Auxiliary telesopes (ATs), and the facilities which are necessary to coherently combine the light for interferometric observations. The interferometric mode is referred to as VLT Interferometer or VLTI. Figure 1 shows the Paranal site as it appeared in April 1995, viewed approximately from the east. The four Unit telescopes will be located in a semi-circle on the rear ridge, the foundations of telescopes 1 through 3 are seen in the background, the foundation of telescope 1 to the left is already finished. Telescope 4 will be located in the pit seen at the lower right edge. The rectangular structure near the centre is the beamcombination laboratory. The half-completed structure going diagonally from the top left to the right is the delay line tunnel. At the time of this writing, progress from the status shown here has been considerable. Projected baselines of the array between Unit telescopes range from $20 \mathrm{~m}$ to $132 \mathrm{~m}$, those between Auxiliary telescopes range between $4 \mathrm{~m}$ and $200 \mathrm{~m}$. These numbers include baseline foreshortening due to Zenith distances of up to 60 degrees. The resulting resolution approaches 0.5 milli-arcsec at visible wavelengths and 1 milli-arcsec in the near infra-red.

The concept of VLTI is shown in fig. 2. Light from the coude foci of the Unit and Auxiliary telescopes is fed into a system of underground light ducts and enters the $160 \mathrm{~m}$ long, $8 \mathrm{~m}$ wide delay line tunnel. Cat's eye sys- 
tems move along $60 \mathrm{~m}$ benches to provide the optical delay needed to cancel the geometric path differences which occur when sources are observed off zenith. Beam combination instruments are set up in the central laboratory facility.

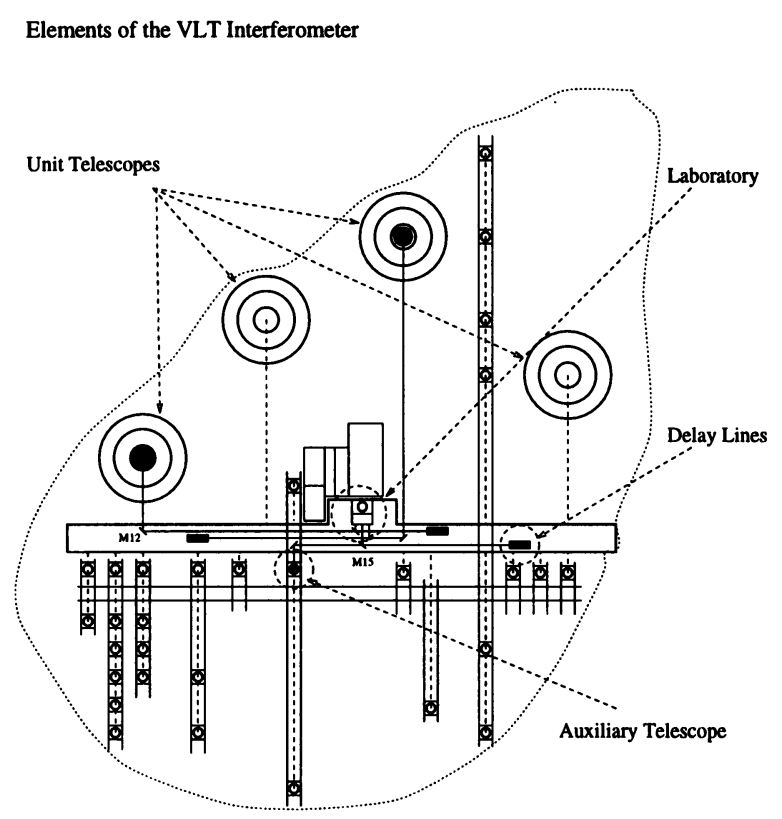

Figure 2. Concept of the VLTI.

One of the main science targets of VLTI is stellar physics, we have already presented some science goals in an earlier paper (von der Lühe et al. (1995)). The surface of many stars can be mapped with a resolution of better than a dozen pixels across the stellar surface. We discuss here the number of stars for which this will be possible, and the nature of phenomena which we expect to observe.

High angular resolution permits detailed studies of the structure of stellar atmospheres, their hydrodynamics and magneto-hydrodynamics. Although target stars are usually bright, the large collecting area of VLTI is essential for measuring the faint fringes resulting from the resolved stellar surface at spectral resolution high enough to resolve spectral line features. In order to better understand the requirements, we are in the process of developing detailed models of stellar surfaces to simulate observations with VLTI. We will present first results in this paper. 


\section{Interferometry of stellar surface structure}

\subsection{POTENTIAL SOURCES}

The prime observables of a stellar interferometer are the contrast of the fringes produced by each telescope pair and their relative position (phase). Both quantities are essential in obtaining resolved surface maps. Each telescope pair produces a fringe across the image of a star at the coherent focus, the angular frequency of the fringe depends on the observing wavelength and the projected baseline. Fig. 3 shows two elements, 1 and 2, of the array which are separated by the baseline $\vec{B} I_{12}$ (solid arrow) and the baseline projected towards the source $\vec{B} \prime_{12}$ (dashed arrow). If $\vec{B} \prime_{i k}$ denotes the projected baseline between telescopes $i$ and $k$ and $\bar{\lambda}$ denotes the mean observing wavelength, then the angular frequency $\overrightarrow{i_{i k}}$ of the resulting fringe, measured in line pairs per radian, is given with

$$
\overrightarrow{s_{i k}}=\frac{\vec{B} \prime_{i k}}{\bar{\lambda}} \quad \operatorname{lp} \operatorname{rad}^{-1}
$$

For optical interferometers with typical baselines of $100 \mathrm{~m}$ and at wavelengths of the order of $1 \mu \mathrm{m}$, angular frequencies are typically $10^{8} \mathrm{lp} \mathrm{rad}^{-1}$, which corresponds to fringe spacings of two milli-arcsec. VLTI's baselines provides for angular resolution in the range of $1.5 \ldots 10$ milli-arcsec for UTs, and of $1 \cdots 50$ milli-arcsec for ATs.

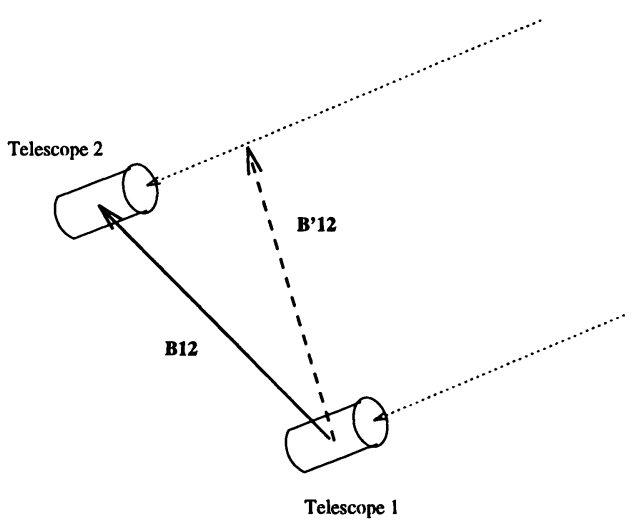

Figure 3. A two element interferometer.

Apparent stellar diameters must be at least a few times the minimum fringe spacing achievable with a given interferometer in order to be resolvable. VLTI will therefore be capable to obtain a few fundamental parameters of stellar structure, such as diameters and center-to-limb variation, for 
stars with diameters of the order of a milli-arcsec. Detailed surface structure will be observable for stars with diameters in excess of 5 milli-arcsec.

We have used the Catalogue of Apparent Diameters and Absolute Radii of Stars (CADARS; Fracassini et al. (1988)) to investigate the potential of VLTI for resolving stellar surfaces. This catalogue is available through STARCAT and provides a collection of known apparent stellar diameters at various wavelengths, either through direct techniques (interferometry, occultations, eclipsing binaries and pulsating stars) or indirectly through intrinsic brightness and colour. The vast majority of the data are results of indirect techniques and should therefore be regarded with some caution. We estimate that the catalogue data should be within a factor of two or three from the truth, sufficient to assess the order of magnitude of potential targets for VLTI.

Fig. 4 presents a histogram of the diameter distribution of all sources in the catalogue with an apparent diameter larger than a milli-arcsec and a declination of less than 40 degrees, which is within reach from Cerro Paranal with VLTI baselines. There is a total of 1309 such sources. The figure shows cumulative histograms for different spectral types, as well as the overall cumulative distribution. Virtually all stars which are potentially resolved by VLTI are late types, in particular K Giants. We will be able to measure diameters on only a few early-type stars, and by the time VLTI will be operational this may already have been done with the Sydney University Stellar Interferometer (SUSI; Davis et al. (1994)) or other long baseline interferometers.

About $50 \%$ of all stars shown have apparent diameters of less than 2.5 milli-arcsec; little more than just estimating diameters and CLVs will be possible for those. About $20 \%$ or some 260 are larger than 5 milli-arcsec, it should be possible to produce fairly detailed maps, with a resolution of at least 5 by 5 pixels. Only $7 \%$ or some 90 stars, and all of them $\mathrm{K}$ and $M$ stars, are larger than 10 milli-arcsec, and are already resolved by the VLT Unit telescopes at visible wavelengths. Detailed surface maps will be possible for those stars provided that good baseline coverage is achieved. VLTI has a particular edge here, because there are four big telescopes which can be combined with relocatable auxiliary telescopes.

Fig. 5 shows a scatter diagram of the angular diameters vs. visual magnitude of the selected CADARS sources. Crosses represent indirect measurements while heavy dots, which comprise only some $15 \%$, represent direct measurements. The catalogue is evidently not complete, stars with $m_{V}>6.5$ are underrepresented. It is also seen that there are several diameter estimates for the same magnitude; those represent the same source measured with different techniques at different wavelengths. The main conclusion here is trivial, but important: resolved stars are bright. There are 


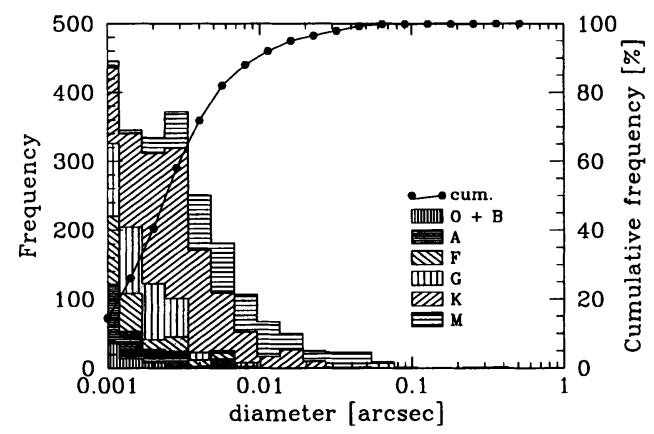

Figure 4. Histogram of CADARS stars with apparent diameters larger than 0.001 arcsec and declinations below 40 degrees.

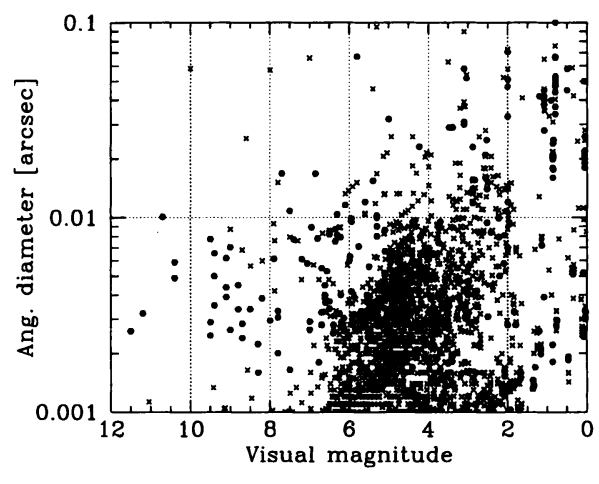

Figure 5. Apparent angular diameters vs. visual magnitude of CADARS stars with declinations below 40 degrees. Solid dots: direct techniques, crosses: indirect techniques.

no stars with visual magnitudes above 12 in the sample, the bulk has magnitudes between 2 and 7 . These sources can therefore serve as their own reference sources for adaptive optics which will perform near the diffraction limit at near infrared - and at later stages of the observatory's development also visible - wavelengths. This circumstance makes VLTI very sensitive for measuring low visibilities. Because of the bias towards brighter stars one can expect many more stars, about a factor 0.5 to 1 of the present sample, at magnitudes above 6.5 and with apparent diameters less than 10 milliarcsec. These will be predominantly $\mathrm{K}$ and $\mathrm{M}$ stars. In total, one can expect about 2000 stars to be resolved with VLTI, out of which some 400 will be large enough to obtain maps of surface detail. 


\subsection{POTENTIALLY OBSERVABLE PHENOMENA}

At the present stage of knowledge, surface phenomena are related to hydrodynamic (e.g. pulsations, convection) and magneto-hydrodynamic effects (e.g. starspots) in the outer layers of stars. In order to assess the magnitude of the interferometric signal expected to be observed with the VLTI, we use the Sun as a proxy for late-type stars with low activity levels. We also assume here that the surface phenomena of an evolved star with higher levels of magnetic activity are qualitatively similar to those of the sun (i.e. there are convective cells, starspots and active regions). The magnetic features, however, are correspondingly bigger for more active stars. The question we ask in this context is what the visibility function, i.e., the normalized Fourier transform of the expected intensity distribution, would be like. The results are presented in figs. 6 (solar-type star) and 7 (giant), which show the various contributions to visibility magnitude. Due to the vast range in both angular frequencies and visibility magnitude, the figures show log-log plots. In order to be independent of assumptions about the distance to the star, angular frequencies are shown in "inverse stellar radii"; however, the feature scale in arcsec shown on the top of the plots illustrate the scales which result from placing the Sun (Fig. 6) and a K giant with 25 solar radii (Fig. 7) at a 10 pc distance.

We consider the following phenomena in these figures:

1. Stellar disc - the disc itself produces the bulk of the interferoemtric signal at low angular frequencies. The reason is that despite the effects of limb darkening, there is always a sharp stellar edge. Fig. 6 shows the Airy function-like visibility of a disc with a limb darkening coefficient of 0.32 . The visibility at higher frequencies is indicated as the dot-dashed line with a high frequency turn-off related to the pressure scale height in the photosphere. The stellar disc component in Fig. 7 is shown for a limb darkening coefficient of 1.0 .

2. Star spots and active regions - both spots and plages would contribute signals to the low to medium frequency range. The visibility contribution shown in Fig. 6 represents five starspot groups, each with a diameter of $2 \%$ of the stellar diameter and a rest intensity of 0.05 times the disc center intensity. The dotted lines represent a variation of the signal by a factor of $\pm \sqrt{5}$. Spots with a diameter of $10 \%$ of the stellar diameter and a rest intensity of $40 \%$ were assumed for the evolved star. Lower spot contrasts on giants are expected due to the larger pressure scale heights and the lower magnetic field strengths. Observations support these expectations (Saar et al. (1995)).

3. Small-scale magnetic fields - the solar surface is dotted with magnetic field concentrated in small elements which manifest themselves as net- 
work bright points (NBPs). According to Auffret et al. (1991) there are roughly $2.3 \cdot 10^{4} \mathrm{NBPs}$ on the solar surface at any given time. Their diameter is less that $100 \mathrm{~km}$ and their contrast can be of order 1.5 times the mean intensity (von der Lühe (1994)), although average values appear to be lower (e.g. Solanki (1992a)). The contribution shown in Fig. 6 assumes the same number of NBPs with a contrast of 1.6 and a $50 \mathrm{~km}$ diameter. There is no such contribution for the evolved star within the range covered by the plot in Fig. 7 .

4. Convection - the contribution shown in Fig. 6 represents solar granulation with an rms contrast of 0.15 times disc central intensity, and with a high frequency cutoff taken from von der Lühe and Dunn (1987). The upper cutoff frequency corresponds to a mean granular size of $1400 \mathrm{~km}$. The lower cutoff was arbitrarily set to 10 times the upper cutoff. The plotted visibility is possibly somewhat optimistic, since we have not taken the reduced granular contrast near the limb into account.

Little is known about the properties of convection for stars of types much later than the sun, but see Nordlund and Dravins, (1990), and Gray, (1988), for convective properties of K stars. Dwarfs show increasingly lower convective velocities (Gray (1988)) and brightness contrast towards later spectral types (Nordlund and Dravins, (1990)). Convective properties of giants are largely unknown, although line broadening velocities increase rapidly with increasing stellar radius (e.g. Gray, (1988)). The radiative transfer in the photospheres of giants is too little understood (Gustaffson et al. (1994)) to determine the contrast of their surface convective cells.. Therefore, we show in Fig. 7 the range of visibility signal one would expect for an intensity contrast varying from $1 \%$ to $25 \%$ for convective cells ten times the size of solar granules, to account for the increase in size with decreasing surface gravity (see, e.g., Nordlund and Dravins, (1990), and Schüssler,(1992)).

It is evident from Fig. 6 that observation of surface phenomena on late dwarfs with VLTI is next to hopeless. The disc of the Sun at 10 pc would be just barely resolved by VLTI baselines, and the variation of stellar radii for late type dwarfs is only within a factor of 3 (Wesselink (1969)). Spots and active region on these low activity stars just produce too little variation in visibility to be measurable. Convection is only accessible to baselines of order of several to several tens of kilometers - unfortunately the top of Paranal is far too small to accomodate those. We note in passing that, although interferometers would be able to sample only a few points in the Fourier domain - which implies that it is utterly hopeless to attempt to image the temperture variations - there is nevertheless quite a bit of interesting science to do. One would be able to probe the statistical properties of surface convection on a number of stars and potentially detect anisotropy 


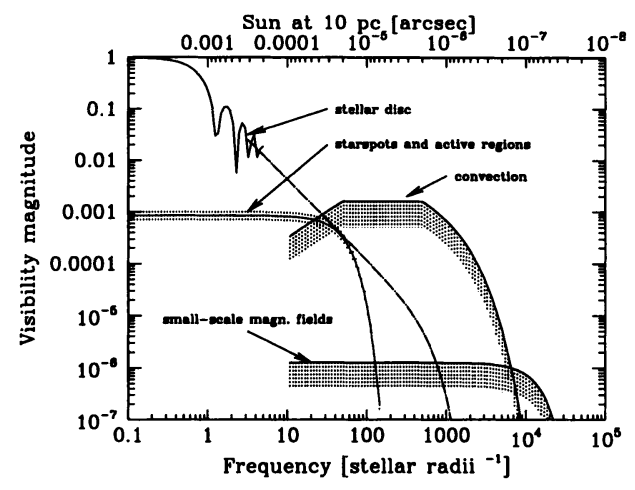

Figure 6. Visibility magnitude for a solar type star.

of the temperature distribution in the photospheres. Correlating those with the orientation of the rotation axis would provide insight in the interaction of Coriolis forces with convection for rapidly rotating stars whose magnetic activity level is orders of magnitude larger than the sun's. The detection of the giant spots which such stars may possess (10 times larger than sunspots in linear dimensions) may begin to become feasible on such dwarfs with VLTI.

With all due reservation because of the assumption made, the situation looks much better for active stars. Starspots and active regions of the sizes implied by Doppler imaging should be easily observable with VLTI on a number of stars. Interestingly, the ideal stars for VLTI are not the same as the ideal targets for Doppler imaging. Earth-rotational synthesis interferometry, the technique which makes VLTI capable of resolving a complex structure, prefers targets which do not change their appearance significantly during one night, while Doppler imaging requires stars with large $v \cdot \sin i$, and consequently large rotatonal surface velocities and high rotation rates. On the other hand, interferometry is insensitive to the orientation of rotation axes and can therefore observe a larger sample of stars. Doppler imaging yields good maps only for inclination angles in the range of $30 \ldots 60$ degrees, thereby limiting the sample to some $40 \%$ of all possible orientations.

Whether or not convection can be observed on giants with VLTI remains to be seen - too little is known about the photosphere of these stars. The presentations made at this conference about surface features on $\alpha$ Ori with $4 \mathrm{~m}$ class telescopes and even the $2.4 \mathrm{~m}$ HST seem to be rather encouraging. 


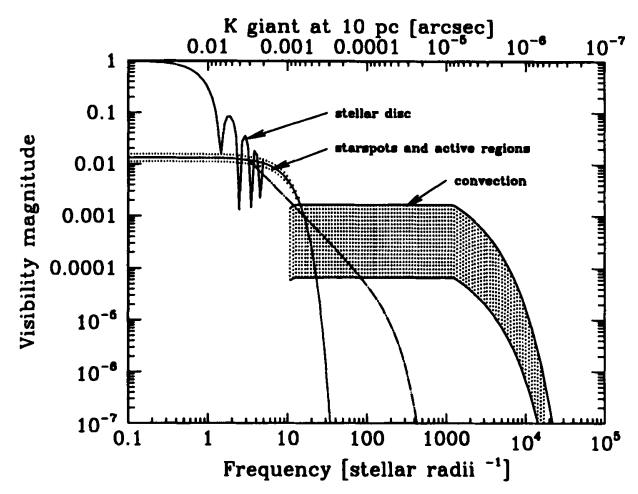

Figure 7. Visibility magnitude for a giant star.

\section{Models of observations with VLTI}

\subsection{OBJECTIVES AND BASIC ASSUMPTIONS}

The study of stellar surface structures will be a key science programme for interferometers which, like VLTI, are optimized for imaging. The demands of such complex sources, which are characterized by low magnitude, complex structured visibility functions, on the operation of the interferometer are quite severe. We are in the process of developing detailed computer models of stellar surfaces to better understand the performance limitations of VLTI, and to assess the difficulties which we are likely to encounter with real observing programmes. The goal is to merge the model soure generator with the output of a separate technical computer model of VLTI, describing the effects of atmospheric and instrumental disturbances, in a physical model which will be used to generate simulated data for further studies. Those will enable us to determine requirements on VLTI performances such as sensitivity to low visibility, the UV coverage as a function of source declination, the spectral resolution and polarimetric precision. We will also better understand the related operational requirements, like how to schedule observations in an efficient manner and how to calibrate data. Of course, simulated observations will allow us to study data processing techniques and to compare the results with the input models for fidelity.

Fig. 8 shows a diagram of the current implementation. A number of source parameters determine the stellar models. Geometric parameters include stellar diameter, ellipticity of an oblate ellipsoid aligned with the axis of rotation, the inclination angle, and the stellar longitude pointing towards the observer. The position and the extent of simple surface features of elliptical shape can be freely specified. Each feature is characterized by its spectral distribution as a function of center from the apparent stellar disc 


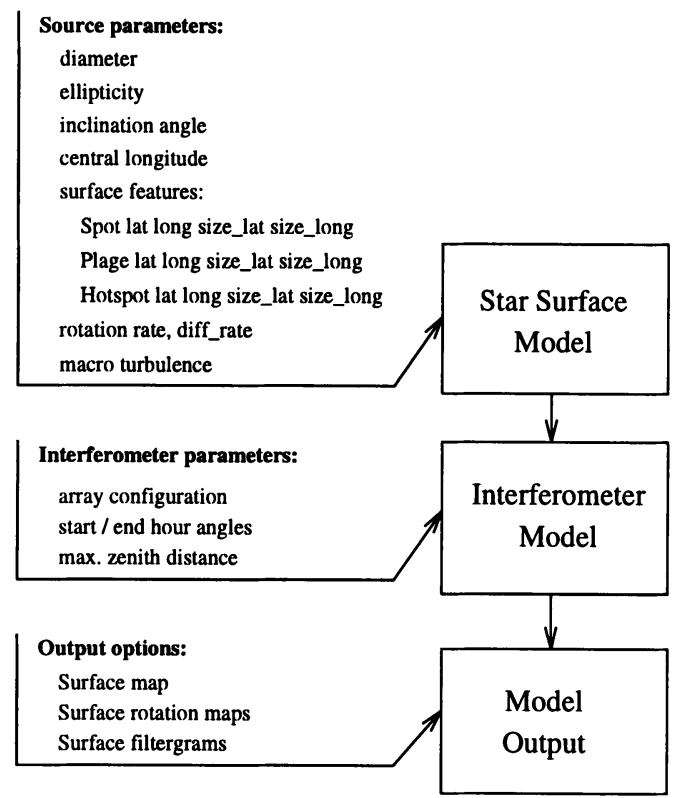

Figure 8. Stellar surface model structure.

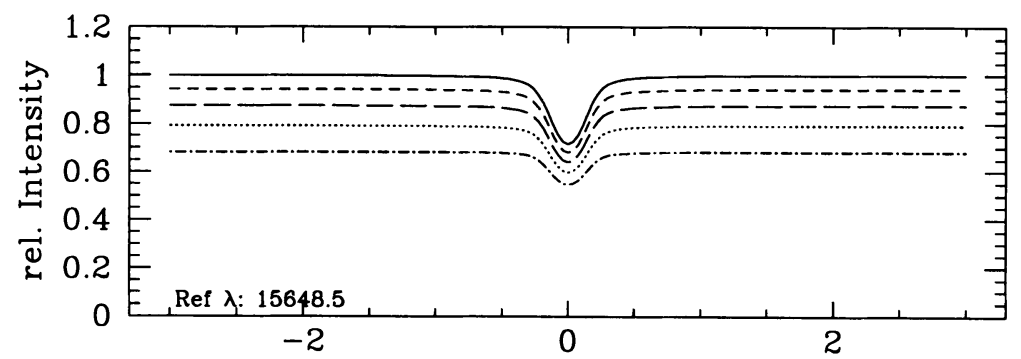

rel. wavelength

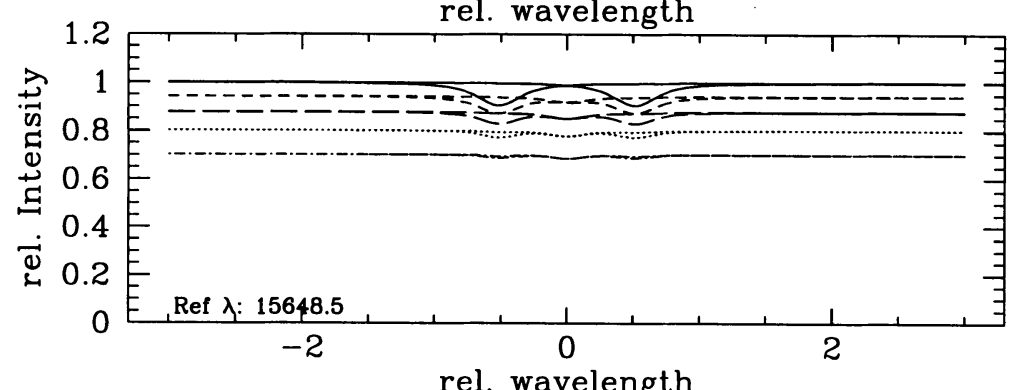

rel. wavelength

Figure 9. Spectra used for model generation. Top: quiet star spectra for, from above, $\mu=1,0.8,0.6,0.4,0.2$. Bottom: active star spectra in right and left circular polarisation, same range for $\mu$. The wavelength scale is relative to the reference wavelength 1.56485 $\mu m$. 
centre. We presently only use spectra which characterize the quiet stellar surface and magnetized plasma to simulate spots, but the hooks to also include plages (magnetized plasma with different radiation characteristics than spots) and as yet unspecified hotspots exist. Fig. 9 shows the sets of spectra at near-IR wavelengths in the vicinity of $\lambda=1.564 \mu \mathrm{m}$ which were used in the following simulations. This spectral region includes a $\mathrm{Fe}$ I line with a Landé factor of 3 (Solanki et al., (1992b)). The upper part shows the spectra for the quiet stellar surface without magnetic field at $\mathrm{T}$ $=5000 \mathrm{~K}$, the lower part shows spectra in left and right circularly polarized light at $\mathrm{T}=4000 \mathrm{~K}$ with a magnetic field of $1.5 \mathrm{kG}$. Spectra are shown for $\mu=\cos \theta$ values of $1,0.8,0.6,0.4$, and 0.2 . The intensity at any radial distance from the disc centre in the models is taken from the spectra by linear interpolation in $\mu$.

The stellar rotation rate and differential rotation, following a simple sine-of-latitude-squared law, are included as well to properly account for line shifts across the stellar disc. Finally, macroturbulence is taken into account by convolution of the spectra with an appropriate Gaussian distribution.

Figure 10 shows a sample star surface, a K0III star with three visible spots, generated by the model. Figure 11 shows three decades of the visibility magnitude of the model in fig. 10 in a logarithmic intensity scale. The result for a star without spots would look similar, only the Airy-like rings would be perfectly circular. Detecting spots on a stellar surface with VLTI is therefore equivalent to detecting deviations from circular symmetry in the visibility function, as well as detecting deviations of visibility phase from 0 and 180 degrees.

The interferometer is only capable to measure the visibility function at angular frequencies which correspond to the projected baselines and the observing wavelength, as shown in eqn. 1. Earth rotation will change the length and the orientation of projected baselines, so the region in the UV domain scanned by the array can be quite substantial when the range of hour angles is large. Fig. 12 shows the UV coverage of VLTI with all four Unit telescopes and three Auxiliary telescopes, for a source at -30 degrees declination and for a full night of observation.

The desired model output can be specified. To date, a \pm 90 degrees latitude, 360 degrees longitude map of the surface can be produced for reference purposes. Sequences of pictures showing adjustable phases of rotation of the stellar surface in continuum light can also be generated at any resolution. Furthermore, simulated filtergrams can be calculated, scanning the available spectral range with a specified bandpass, and for a given orientation of the stellar surface. Of course, the filtergrams correctly represent the Doppler shifts in wavelength across the disc due to the rotation law. The filtergrams are used to generate spectrally resolved fringe patterns. 


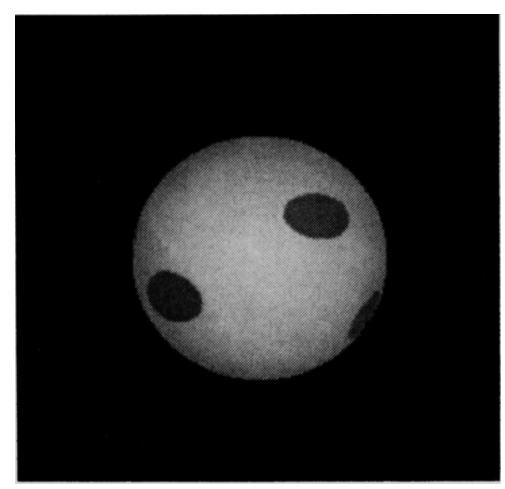

Figure 10. Model of a K0 III surface.

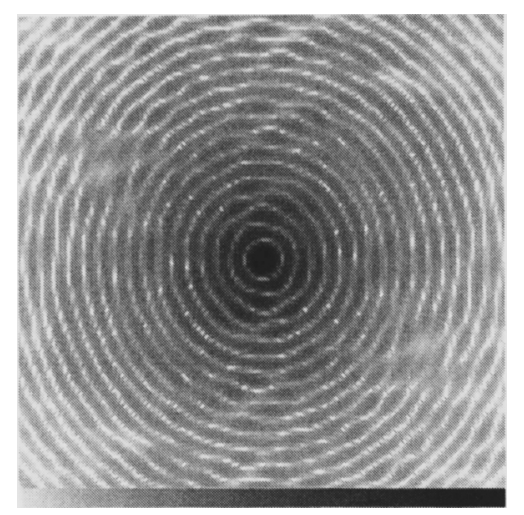

Figure 11. Visibility magnitude of KOIII surface model. Three decades are shown with a logarithmic density scale.

\subsection{INTEGRATED LIGHT SIMULATIONS}

The surface maps generated as described above were used to simulate VLTI in a way described earlier by one of us (Reinheimer et al., (1993)). The interferometer is implemented with an "imaging beamcombiner", where interference takes place in a superimposed focal plane conjugate on top of an image of the source. Because of the source brightness and the wavelength, photon noise was neglected. Seeing was accounted for by assuming partial adaptive optics correction with an effective Fried parameter $r_{0}$ of $2 \mathrm{~m}$. This way, images from individual telescopes are somewhat affected by atmospheric turbulence, but are dominated by a single, bright speckle. No effort was yet made to account for incomplete coherencing and the resulting loss in fringe contrast; such losses could be overcome by choice of a suitably narrow bandpass, which implies a long coherence length. Also, our 


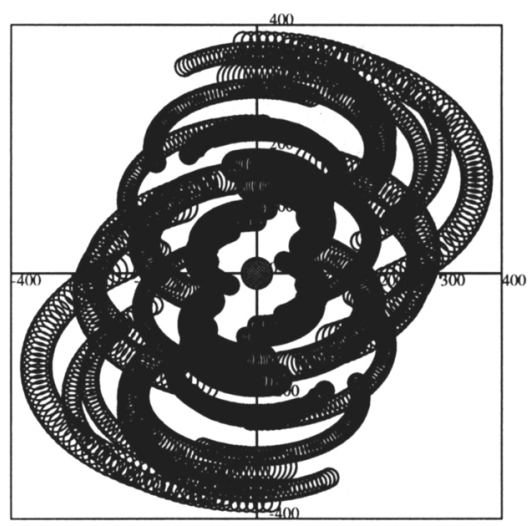

Figure 12. UV coverage of VLTI with four UTs and three ATs, for a source at $\delta=-30$ degrees. The units are line pairs per arcsec for $\lambda=1.56 \mu m$.

present capability does not yet account for any instrumental fringe contrast efficiency (measured fringe contrast divided by theoretical fringe contrast). Our current error budgets show an overall fringe contrast efficiency of better than $50 \%$ with partial adaptive optics being the dominant error source, so instrumental effects should be accounted for within a factor of two. We do not consider this a serious problem; the main limiting factor for generating stellar surface maps will in our opinion be incomplete UV coverage, which is included in the present capability.

A source corresponding to a star with an apparent diameter of 8 milliarcsec and two spots was placed at $\delta=-60$ degrees. A configuration with all four UTs and three ATs was used. 100 short exposure interferograms were simulated for each of 21 hour angles distributed equally about the meridian, this corresponds to integration times of 21 times a few minutes distributed over the whole night. Fig. 13 shows two sample interferograms, one for an unresolved point source (left), the other for the resolved disc (right). Triple correlations of the interferograms were calculated, their Fourier transforms were averaged in a bispectrum with 1182894 elements. After calibration with the bispectrum of a point source, the bispectrum was used to reconstruct the source with 2000 iterations of the building block technique. This analysis process corresponds in general to standard speckle imaging techniques. Fig. 14 shows the simulated source (right), blurred to the angular resolution of the maximum VLTI baseline, and the reconstructed source (right). Except a sub-pixel mutual shift, there is hardly any difference seen between the original and the reconstruction, artifacts appear off the stellar disc at a level of less than $1 \%$ peak intensity.

We take these results as a proof of concept and as an encouraging indication that resolving stellar surfaces will be possible with VLTI. Of course, 


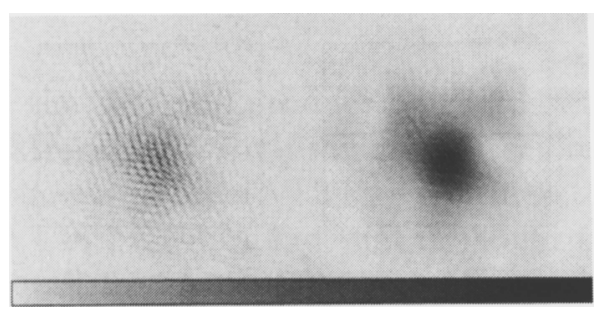

Figure 13. Simulated image plane interferograms, point source (left) and resolved stellar disc (right).

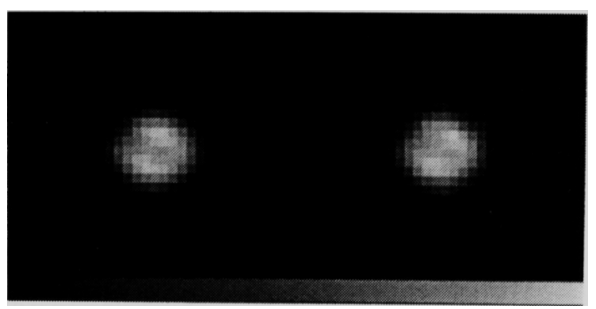

Figure 14. Simulated stellar disc at VLTI resolution (left) and reconstructed stellar disc (right).

a positive result for one example does not imply overall success, and many further studies with different source models at different latitudes, and a better representation of instrumental deficits are needed, and will be performed.

\subsection{SPECTRALLY RESOLVED SIMULATIONS}

The combination of angular resolution with spectral resolution within narrow spectral bands will provide important diagnostics. Quiet and active regions on the surfaces of active stars will differ in their spectral characteristics; corresponding signatures should be detectable in plots of the visibility as a function of wavelength, across suitable spectral lines, at a given baseline. These signatures will depend on the number of spots and on their location, thus providing valuable independent information in addition to the variation of visibility observed in the continuum. Because of generally incomplete UV coverage and the complexity of the sources, these spectral signatures may be the only way to obtain unambiguous results. 

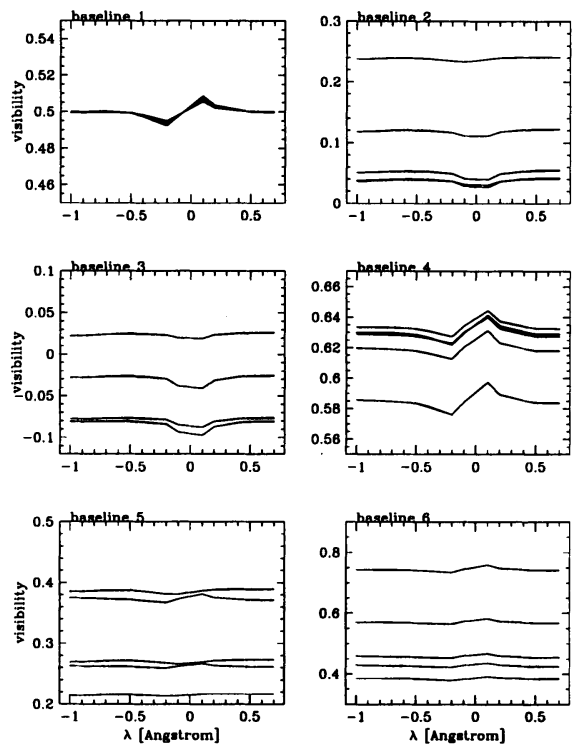

Figure 15. Simulated variation of the real part of complex visibility with wavelength. Each panel represents one of the six baselines of the VLTI main array. The five plots within each panel represent "visibility spectra" at hour angles of $-60,-30,0,30$ and 60 degrees for a $\delta=-35$ degrees source.

Furthermore, it is likely that visibility spectra will be "self calibrating" - $i$. e., the visibility data will contain the same errors at all wavelengths. The consequence is that the visibility magnitude may be in error while the details of the signatures are not. This circumstance may add robustness to inversion procedures. At this time, the proper use of spectrally resolved information in interferometry has received relatively little attention (see the contribution by Phillipe Stee et al. in this volume). Inversion techniques to extract information from such data are rare and need to be developed, and we expect that our models will help to do so.

We are just beginning to simulate spectrally resolved observations. As an example, we show in fig. 15 the real part of the complex Fourier transform as a function of wavelength, as it would be measured by the six baselines of the Unit telescope array for a star similar to the one studied in the previous section. Each panel shows the real part of complex visibility as a function of wavelength across the Fe I line. Within each panel, there are five plots corresponding to five hour angles. It is seen that visibility does vary across the line profile, as described above. It is our plan to analyze data of this kind with techniques similar to radio very long baseline image synthesis in the future. 


\section{Conclusions}

Interferometry with the VLT will produce crucial new insight in the understanding of stellar atmospheres by providing direct images of stellar surfaces. By assessing the potential of VLTI we conclude that about 2000 stars will be resolved to VLTI baselines and that it should be possible to obtain maps with useful detail for some 400 stars. A large fraction of the latter are late type giants, which indicates that at least for this class of stars, a fairly complete sample of maps should be doable. Of course, one objective would be to simultaneously observe stars with interferometric and Doppler imaging techniques to compare the results. We expect that the available baseline length will limit the resolution to structures of the order of large spots and active regions on magnetically active stars.

We use simulated stellar surfaces to study in detail the performance of VLTI, to investigate operational requirements of a given science program, and to study data analysis methods. This work has just started. First results shown here indicate that resolving stellar surfaces with VLTI will be feasible; however, much more work is needed to understand the limitations. One aspect we already noted is the importance of the "hybrid mode" of VLTI, i. e., the combination of Unit telescopes with one or several Auxiliary telescopes to improve the UV coverage. Adding ATs to the array of UTs improved the simulated reconstructions in the continuum. This issue also requires follow-up; it is crucial to understand much better quantitatively the limitations in mapping fidelity due incomplete UV coverage.

\section{References}

Auffret, H., Muller, R. 1991, A\&A 246, 264.

Davis, J. 1994, in IAU Symp. 158, Very High Angular Resolution Imaging, p. 135.

Fracassini, M., Pasinetti-Fracassini L. E., Pastori, L., Prioni, R. 1988, Bull. CDS 35, 121. Gray D.F., 1988, Lectures on Spectral-Line Analysis: F, G, and K Stars, The Publisher. Gustafsson, B., Jørgensen, U. G. 1994, ARA\&A 6, 19.

Nordlund, A., Dravins, D. 1990, A\&A 228, 155.

Reinheimer, Th., Hofmann, K.-H., Weigelt, G. P. 1993, A\&A 279, 322.

Saar, S. H., O'Neal, D., Neff, J. E. 1995, in IAU Symp. 176, Poster Proceedings: Stellar Surface Structure, K.G. Strassmeier (ed.), University of Vienna, Vienna, p. 105.

Schüssler M., 1992, in The Sun - a Laboratory for Astrophysics, J.T. Schmelz, J.C. Brown (eds), Kluwer, Dordrecht, p. 81

Solanki S.K., Brigljević V., 1992, A\&A 262, L29.

Solanki S.K., Rüedi, I., Livingston, W. 1992, $A \& A$ 263, 312.

von der Lühe, O., Dunn, R. B. 1987, $A \& A$ 177, 265.

von der Lühe, O. 1994, $A \& A$ 281, 889.

von der Lühe, O., Schüssler, M., Solanki, S., Caligari, P. 1995, Science with the VLT, J.

R. Walsh and I. J. Danziger (eds), ESO Astrophysics Symposia, Springer, p. 94.

Wesselink, A. J. 1969, MNRAS 144, 297. 


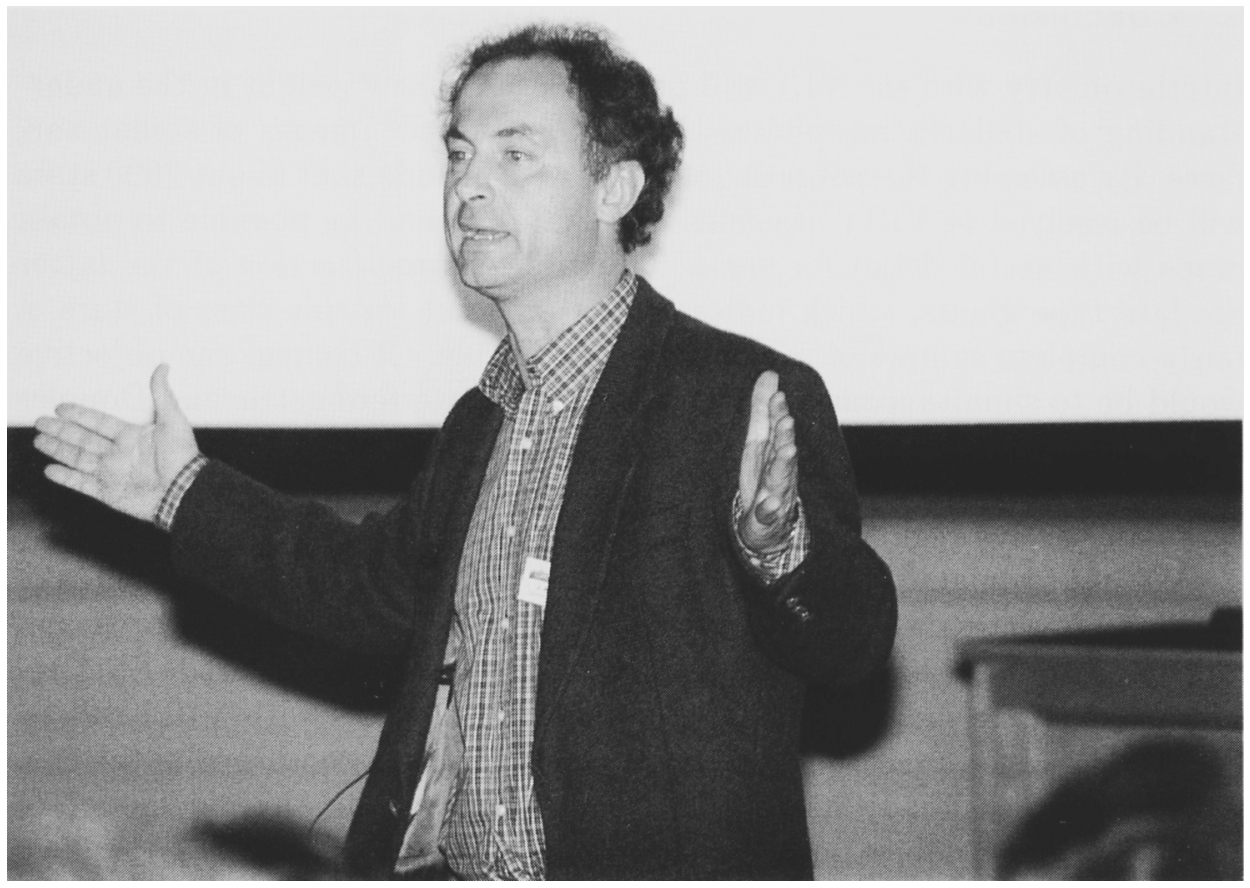

Jean-Francois Lestrade's VLTI baseline (top) seems to be even longer than that of Oskar von der Lühe's (bottom right). Notice that Ron Gilliland usually observes in the ultraviolet (bottom left)!
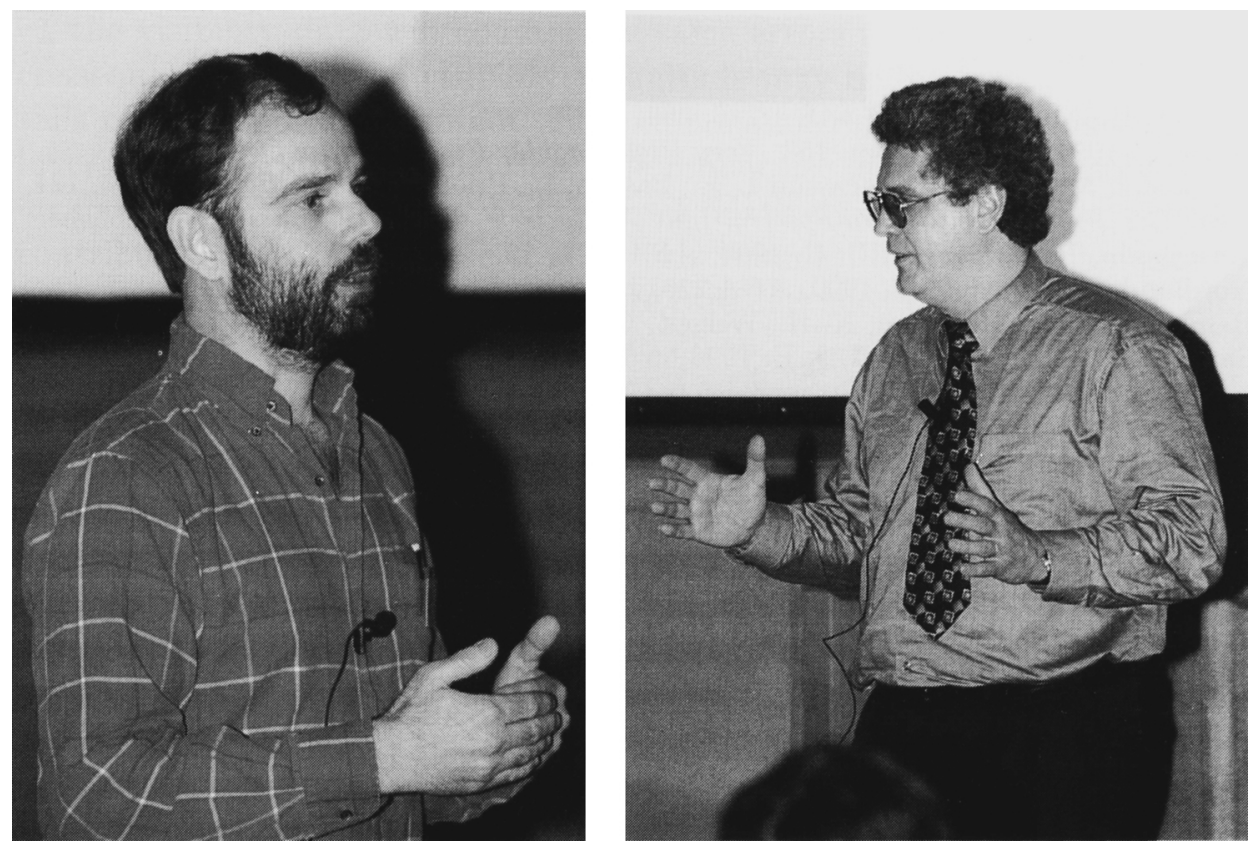